\title{
Anhang.
}

\section{Zur Vorbereitung einer Sammelforschung über Syphilis und ihre Behandlung.")}

\author{
Von
}

Prof. Dr. Heinrich Köbner in Berlin.

Nachdem mein Antrag auf Anbahnung eines, überall vou denselben Gesichtspunkten geleiteten Beobachtungsmodus Syphilitischer und auf Berathung des von mir zu diesem Zwecke, beziehungsweise zur Anbahnung einer Sammelforschung dieser Section vorgelegten Beobachtungsschemas durch eine Commission in der letzten Sectionssitzung der vorjührigen Naturforscherversammlung genehmigt worden war, habe ich im lotzten vorjährigen Hefte der "Vierteljahresschrift f. Dermat. u. Syph." unter der Ueberschrift: "Zur statistischen, bezw. zur Sammelforschung über die Pathologie und Therapie der Syphilis" moiner Generaltabelle und einer dieselbe ergänzenden, nur die Descendenz Syphilitischer betreffenden Specialtabelle nur wenige Worte der Begründnng vorausgeschickt. $\mathrm{Da}$ es mir unmöglich ist, den diesjährigen Sitzungen beizuwohnen und den Bericht der Commission mündlich zu eröffnen, möchte ich zur Motivirung heute noch Nachfolgendes anführen.

Tin Ueberblick über die zahlreichen statistischen Publicationen, und die darauf begründeten Schlüsse über Syphilis und ihre Behandlungsmethode aus den verschiedensten Anstalten lehrt, dass erstens eine grosse, von der Hospital- und polikJinischen Statistik unzertrennliche Fehlerquelle in der viel zu kurzen und frag-

1) Eingesandt und vorgelesen in der Section für Dermatologie und Syphilis der Versammlung deutscher Naturforscher und Aerzte zu Wiesbaden 1887. 
mentarischen Daner der Beobachtungen liegt, zweitens aber das publicirte Material auch qualitativ höchst ungenügend brauchbar ist. Man beobachtet und publicirt bisher nur die in einem bestimmten Zeitabschnitt zur Behandlung gelangten syphilitischen Symptome (d. h. Formen und Perioden der Syphilis) und ihre Beeinflussung, beziehungsweise Recidive nach einer oder der anderen Medication, trägt aber der Qualität der so höchst verschiedenen syphilitischen Individuen keine. Rechnung, obwohl die von der Individualität dieser Kranken abhängigen Factoren auch für den natürlichen, von gar keiner Therapie beeinflussten Verlauf ihrer Syphilis anerkanntermassen böchst wichtig sind. Durch diese einseitige und oberflächliche Beobachtungsweise gehen zunächst für viele, noch offene Fragen der Pathologie und namentlich der Therapie der Syphilis selbst, sowie ihrer Beziehungen zu allen anderen Krankheiten jährlich Tausende von Fällen verloren. Ferner aber fehlt für einen denkenden und forschenden Arzt von vornherein die Berechtigung, das vielleicht von Hause aus höchst verschiedene Krankenmaterial verschiedener Anstalten oder Einzelbeobachter zunächst bezüglich des Heilwerthes der verschiedenen von ihnen angewandten und gerühmten Methoden untereinander zu vergleichen. So mögen, um Beispiele anzuführen, abgesehen zunächst von der Verschiedenheit der Ergebnisse zwischen Männer- und Weiberabtheilungen, namentlich solcher, die sich hauptsüchlich aus Scortis recrutiren, in der einen Anstalt 5 Percent, in einer zweiten 15-20 Percent oder mehr Tuberculose und Serophulose unter den Kranken, in einer dritten ein hoher Percentsatz von Malaria, von Alkoholismus u. dgl., sowie umgekebrt in einer vierten Kranke mit ungewöhnlich günstigen allgemein-hygienischen Verhältnissen vorhanden sein.

Es lenchtet nun ein, dass durch die Annahme derselben Grundsätze bei der Untersuchung der Syphilitischen in erster Reihe unter den Specialisten selbst eine Fülle soliden Materials, sowie eine bisher nicht geübte Gleichmässigkeit der Beobachtungsweise und demzufolge Vergleichbarkeit der Ergebnisse ohne Weiteres zu ermöglichen ist.

Durch überall erfolgende methodische Eintragung der gröbsten individuellen Momente, z. B. des Körpergewichts und 
der Constitution, erheblicher anderweitiger Krankheiten, sämm tlicher Organbefunde - auch derjenigen Organe, auf welche keine groben Symptome binleiten - sowie auch der früheren Infectionen würde ausser dem Gewinn in der angedenteten pathologischen Hinsicht namentlich auch die Schwere jedes einzelnen Falles für die Therapie sich in objectivster Weise erkennen lassen, durch methodische genaue Eintragung der Arzneimengen und der Dauer jeder früheren und gegenwärtigen Cur eine Anzahl weiterer therapentisch wichtiger Fragen schon viel rascher und überzeugender lösen lassen.

Bei der bekannten Thatsache des vielfachen Wechselns und vorzeitigen Verschwindens der Symptome aus der Beobachtung jedes Einzelnen und jeder Anstalt muss aber (zur Beseitigung der sub 1 genannten Fellerquelle) auch die Mitwirkung sowohl aller anderen, in späteren Stadien von ihnen aufgesuchten Kliniken und Abtheilungen, als nameutlich der Familienärzte dringend erbeten werden, um eine grosse Zahl langjühriger Beobachtungen Syphilitischer bezüglich ihrer Recidive und bezüglich ihrer Descendenz zu sammeln.

Die Commission hat sich diesen Gesichtspunkten angeschlossen und für die Sammelforschung die zwei, in dieser unserer und allen anderen klinischen Sectionen der heutigen Naturforscherversammlung vertheilten Tabellenformulare als Grundlage angenommen, in deren erste alle Individuen sowohl mit acquirirter als mit hereditärer Syphilis, in die zweite $\mathrm{nur}$ die Uebersicht über die gesammte Familio (Nachkommen) syphilitischer Eltern mit Rücksicht anf deren Behandl ung einzutragen ist. Die Commission setzt voraus, dass dieselben Fragen, welche in den Colonnen der Tabelle I behufs Zusammenstellung der Einzelergebnisse übersichtlicher neben einander gedruckt sind, für die Einzelaufnabmen der Kranken bequemer unter einander (als einheitliche Krankengeschichte, aus welcher die wesentlichsten Rubra in unsere Tabelle zu übertragen sind) beantwortet werden. Unter den Hauptüberschriften: Nationale, Anamnese, Stat. praes., Therapie und Decursus auf jedem Einzelformulare sind dieselben zu subsummiren, überdies noch unter "Anamnese" eine Frage nach der Infectionsquelle (Confrontatiou) 
und für Fälle von vererbter oder hereditärer Syphilis die in Tabelle II enthaltene Fragengruppe mit aufzunehmen.

Ich hatte auch ein derartiges Formulare ausgearbeitet, von welchem ich jedoch, da eine Einigung nicht zu Stande kam, keine Abdrücke anfertigen liess; eine Abschrift davon liegt hier bei. Anschliessend an dasselbe haben die Collegen Pick und Neisser ihre bisherigen Einzelformulare abgeändert und vertheilen Abdrücke derselben hier als Beispiele. Ein Doppelformular des Collegen I. Neumann, das für hereditäre und acquirirte Syphilis einen getrennten anamnestischen Fragebogen enthielt, hat so wenig wie die vorstehenden die einstimmige Zustimmung der Commission gefunden.

Die Commission überlässt die Aulegung von Formularen fụ̈ die Einzelaufnahmen allen Anstalten und Einzelbeobachtern selbst, da ja unsere Tabellen zugleich als Fragebogén für die Einzelformulare dienen, überdios aber für einzelne Anstalten Dentschlands sowie Oesterreichs bosondere Verwaltungsnormen bestehen, die einer besonderen Berücksichtigung bedürfen. Für den Bedarf in der Poliklinik, gleichwio in der Privatpraxis reichen übrigens nach meiner persönlichen Erfahrung schon die zwei hier vorgelegten Tabellen in der jetzigen typographischen-Ausführung aus.

Doch werden die anwesenden Commissions-Collegen, welche wenigstens schon einige Monate dieselben praktisch geprüft haben, sich eingehender äussern, und wir erbitten etwaige Aenderungsvorschläge auch aus dem Schosse der Section, insoferne vielleicht einzelne Theilnehmer derselben schon nach meinen ursprünglichen Tabellen gearbeitet haben.

Zwei Hauptfragen in praktischer Beziehung betreffen die Ausführbarkeit und die genügende Sicherheit der objectiven Erhebungen. In Anstalten kann beides leicht erreicht werden durch Theilung der Arbeit. Ich schlage vor, dass auf allen Abtheilungen für Syphilis - so wie ich dies z. B. in meiner Poliklinik durchgeführt habe - eine feststehende Untersuchung aller Kranken durch ophthalmologische, otiatrische und, wo die eigenen Kräfte und Assistenten nicht ansreichen und genügende Bürgschaft für sichere Resultate bieten, anch durch interne Collegen geschehe. Umgekehrt werden die Syphilidologen allen 
¿nderen Anstalten, um deren Mitwirkung wir bitten; bei ihren Aufzeichnungen, so oft sie es begehren, zur Hand sein. Von ihnen allen ist bei Berücksichtignng und jedesmaliger Ausfüllung der die Syphilidologie besonders interessirenden Fragen ein reiches, bisher noch wenig erschlossenes Material zu erwarten.

Anders stellen sich obige Fragen bezüglich des praktischen Arztes. Ob für seinen Bedarf uud die Möglichkeit seiner, uns so werthvollen Mitwirkung ein kürzeres Schema auszuarbeiten, oder ob nur dasselbe in handlicherer Form herzustellen wäre, z. B. in Form einiger zusammengehefteter Blätter in Octavformat, welchen eine genaue Specialisirung der Fragen als Erläuterung vorangedruckt wäre, die der Eintragende immer vor Augen hätte, darüber, sowie über einige andere rein praktische Fragen erbitten wir die Beschlüsse der Section. Solche sind ferner, da eine mündliche Berathung der Commission nicht stattfinden konnte, sondern nur ein die Wissenschafts-Grundfragen umfassender und sich in die Lünge ziehender Briefwechsel: 1. Welche ärztlichen Kreise hält die Section für zweckmüssig anfzufordern? 2. In welcher Form? Soll die Commission $\mathrm{nur}$ unter Voranschickung eines Motivenberichtes die hier zu beschliessendeu Schemata in allen gelesenen, allgemein-medicinischen Journalen Deutschlands und Oesterreichs publiciren und alle Aerzte zur Mitarbeit auffordern o der sollen, was vielleicht wirksamer ist, direct an alle Anstalten und beziehungsweise ärztlichen Vereine jetzt sofort Zusubriften gerichtet werden? Sollen nur Probeexemplare der Tabellen angeboten, resp. mitgeschickt oder dieselben insgesammt gratis zur Ausfüllung und Rïcksendung an die Commission allen Interessenten offerirt werden? Soll die Rücksendung alljährlich verlangt und alljährlich neue Exemplare den Mitarbeitern zugesendet werden?

Ueber alle diese Fragen die Entscheidung der Section erbittend, möchte ich, indem ich von der Function als Obmann der Commission, welche ich wegen anderweitiger Ueberbürdung nicht weiter übernehmen kann, zurücktrete, vorschlagen, zur schleunigen Ausführung der Beschlüsse, speciell der genannten werthen $\mathrm{Zu}-$ schriften, statt einer vielgliederigen Commission nunmehr einem einzelnen, praktisch besonders gewandten jüngeren Collegen das Mandat zu ertheilen. Zur Bearbeitung der Eingänge könnte eine 
kleinere Commission bestehen bleiben. Zur Beseitigung eines nicht unerheblichen Bedenkens, welches die praktischen Collegen zurückschrecken könnte: die Verletzung der ärztlichen Discretion durch Nennung der Namen in den Sammelbogen, möchte ich mit dem Vorschlage schliessen, statt der Namen in Col. 2 nur die Anfangsbuchstaben des Vor- und Zunamens, das Geschlecht, Tag, Jahr und Ort der Geburt, die Haar- und Hautfarbe des Kranken, sowie oben auf jedem Bogen ihren eigenen (der Einsender) Namen anzugeben.

\section{Anmerkung der Redaction.}

Wegen der grossen Wichtigkeit des Gegenstandes haben wir der Zuschrift des Herrn Prof. Köbner an dieser Stelle Raum gegeben.

Die Section hat Herrn Köbner ersucht, die Stelle des Ob. mannes der Commission woiter zu behalten und im Vereine mit den Mitgliedern derselben, den Herren Doutrelepont, Bonn, Lewin, Berlin, Neisser, Breslau, Neumann, Wien, F. J. Pick, Prag, die Angelegenheit fortzuführen.

Wir lassen nun einen Abdruck der vereinbarten Tabellen folgen, donen die Bedeutung von Fragebogen zukommt und bemerken, dass jene Herren Collegen, welche sich an der Sammelforschung betheiligen und von den bereit gehaltenen Formularen Gebrauch machen wollen, letztere jederzeit von den Mitgliedern der Commission beziehen können, sowie jedes Mitglied der Commission sich erbietet, die ausgefüllten Formularien zur weiteren Verarbeitung entgegen zu nehmen.

F. J. Pick. 
Vorbereitung einer Sammelforschung üher Syphilis und ihre Behandlung.
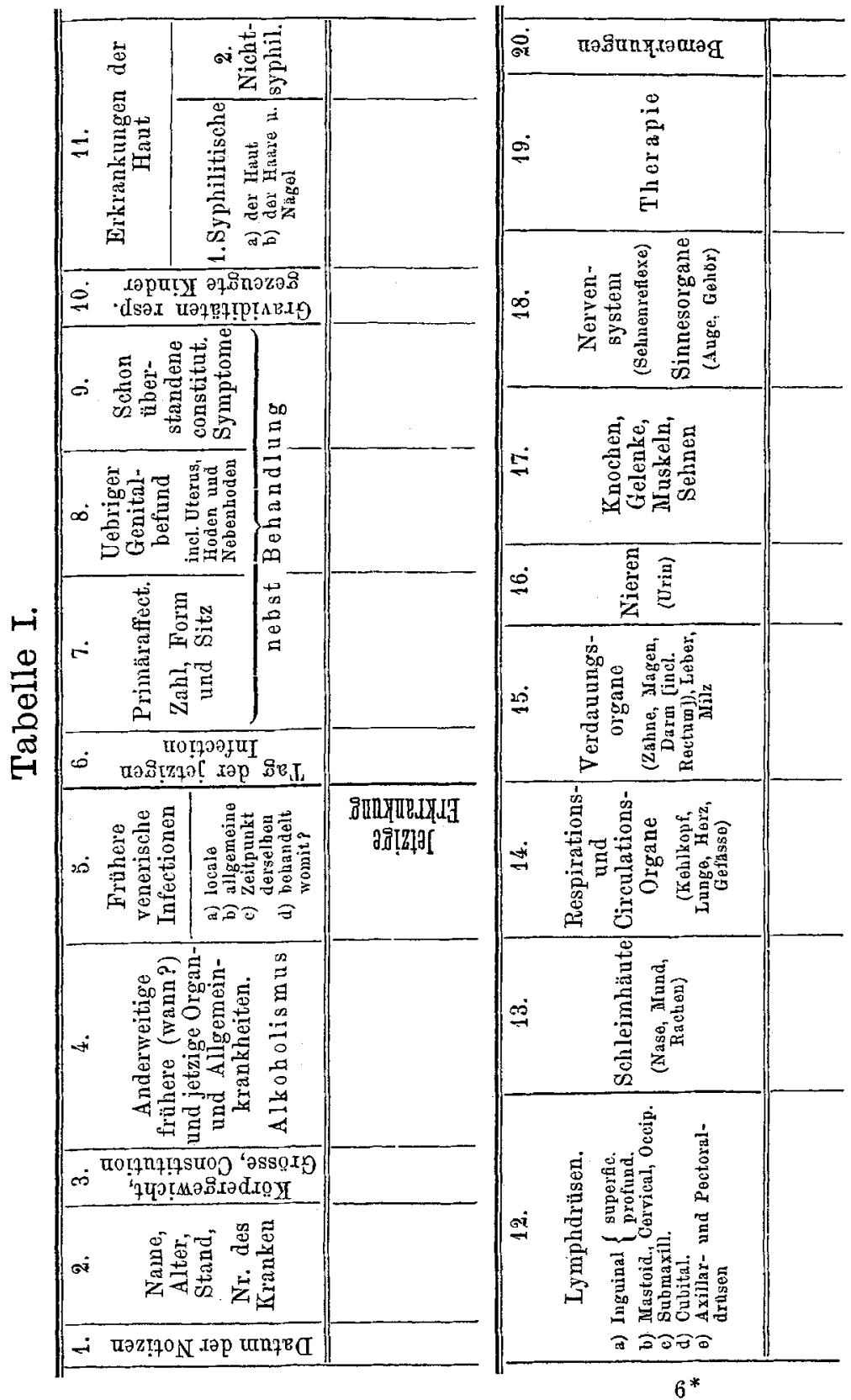
84 K o b ner. Vorbereitung einer Sammelforsclumg aber Syphilis u. ihre Behandl.

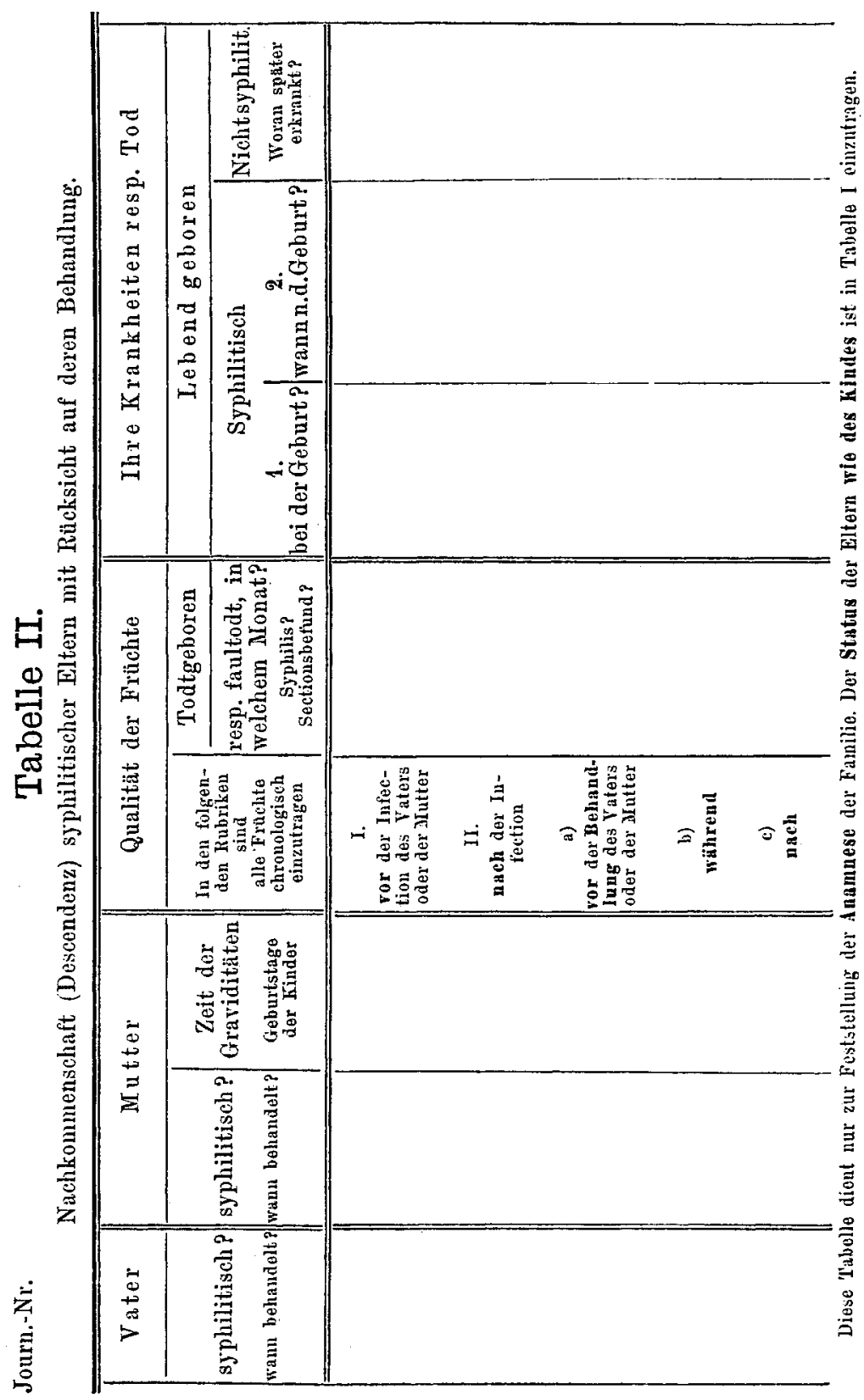

